Review

\title{
Fighting rheumatoid arthritis: Kv1.3 as a therapeutic target
}

\author{
Antonio Serrano-Albarrás, Sergi Cirera-Rocosa, Daniel Sastre, Irene Estadella, Antonio Felipe* \\ Molecular Physiology Laboratory, Departament de Bioquímica i Biomedicina Molecular, Institut de Biomedicina Universitat de Barcelona (IBUB), Barcelona, Spain
}

\section{A R T I C L E I N F O}

\section{Keywords:}

Potassium channels

Kv1.3

Rheumatoid arthritis

Autoimmune diseases

Therapeutics

Pharmacological targeting

\begin{abstract}
A B S T R A C T
Rheumatoid arthritis (RA) is a serious autoimmune disease that has severe impacts on both the wellbeing of patients and the economy of the health system. Similar to many autoimmune diseases, RA concurs with a long evolution, which eventually results in highly debilitating symptoms. Therapeutic treatments last for long periods during RA. However, their efficiency and side effects result in suboptimal conditions. Therefore, the need for specific, safer and nontoxic alternatives for the treatment of RA is essential.

Kv1.3 is a voltage-gated potassium channel that has a crucial role in immune system response. The proliferation and activation of leukocytes are linked to differential expressions of this channel. The evidence is particularly relevant in the aggressive $\mathrm{T}$ effector memory $\left(\mathrm{T}_{\mathrm{EM}}\right)$ cells, which are the main actors in the development of autoimmune diseases. Blockage of Kv1.3 inhibits the reactivity of these cells. Furthermore, pharmacological inhibition of Kv1.3 ameliorates symptoms in animal models of autoimmune diseases, such as experimental autoimmune encephalomyelitis or induced psoriasis with no side effects. Kv1.3 is sensitive to several animal toxins and plant compounds, and several research groups have searched for new Kv1.3 blockers by improving these natural molecules. The research is mainly focused on enhancing the selectivity of the blockers, thereby reducing the potential for side effects on other related channel subunits. Higher selectivity means that treatments will potentially be less harmful. This leads to a lower discontinuation rate of the therapy than the current first-line treatment for RA. The molecular backgrounds of many autoimmune diseases implicate leukocyte Kv1.3 and suggests that a new medication for RA is feasible. Therapies could also be later repurposed to treat other immune system disorders.
\end{abstract}

\section{Introduction}

Autoimmune diseases are common maladies caused by an anomalous function of the immune system. Pathological activation results in immune cells targeting cellular or organ-specific self-antigens. The disease usually progresses to local or even systemic inflammation processes which seriously compromise the homeostasis of the tissue, as well as its function [1]. The consequences of autoimmune diseases are variable as more than 80 have been reported in humans [2].

Some autoimmune diseases, such as multiple sclerosis, RA or myasthenia gravis, cause highly debilitating effects on the wellbeing of the patients [3-5]. Other maladies, such as type 1 diabetes mellitus or celiac disease are equally dependent on pharmacological control [6,7]. Regardless, the total prevalence of autoimmune diseases is up to $4.5 \%$ in the United States [8]. Therefore, autoimmune diseases do not just threaten the wellbeing of millions of people worldwide but also present a high socioeconomic impact.

Autoimmune diseases do not typically trigger acute illnesses; however, their symptoms appear as an accumulation of hidden insults to certain tissues in a chronic manner [9]. During development, $\mathrm{T}$ and B lymphocytes reactive to self-antigens can be produced. These cells tend to be wiped out or functionally silenced early on by a process of overactivation (tolerance) [10]. Nevertheless, these mechanisms fail in diverse ways, generating lymphocytes that should not be reactive.

Several theories claim differential onsets for this event. The cryptic determinant theory states that some antigens or antigen determinants are usually hidden from immune detection [11]. This could be because an antigen is in a remote location (e.g., the eye) or because the typical conformation of a protein normally hides the antigenic determinant. The inaccessibility of the antigen results in self-antigen-reactive lymphocytes, which are not correctly silenced. Other theories support foreign antigens mimicking self-antigens [12], which cause cross-reactivity from the lymphocytes and alterations of the glycosylation profile of the immune system components [13]. The most controversial hypothesis, though, links the high penetrance of autoimmune diseases in developed countries with a higher level of hygiene [14].

\footnotetext{
* Corresponding author at: Molecular Physiology Laboratory, Departament de Bioquímica i Biologia Molecular, Institut de Biomedicina, Universitat de Barcelona, Avda Diagonal 643, E-08028 Barcelona, Spain.

E-mail address: afelipe@ub.edu (A. Felipe).
} 

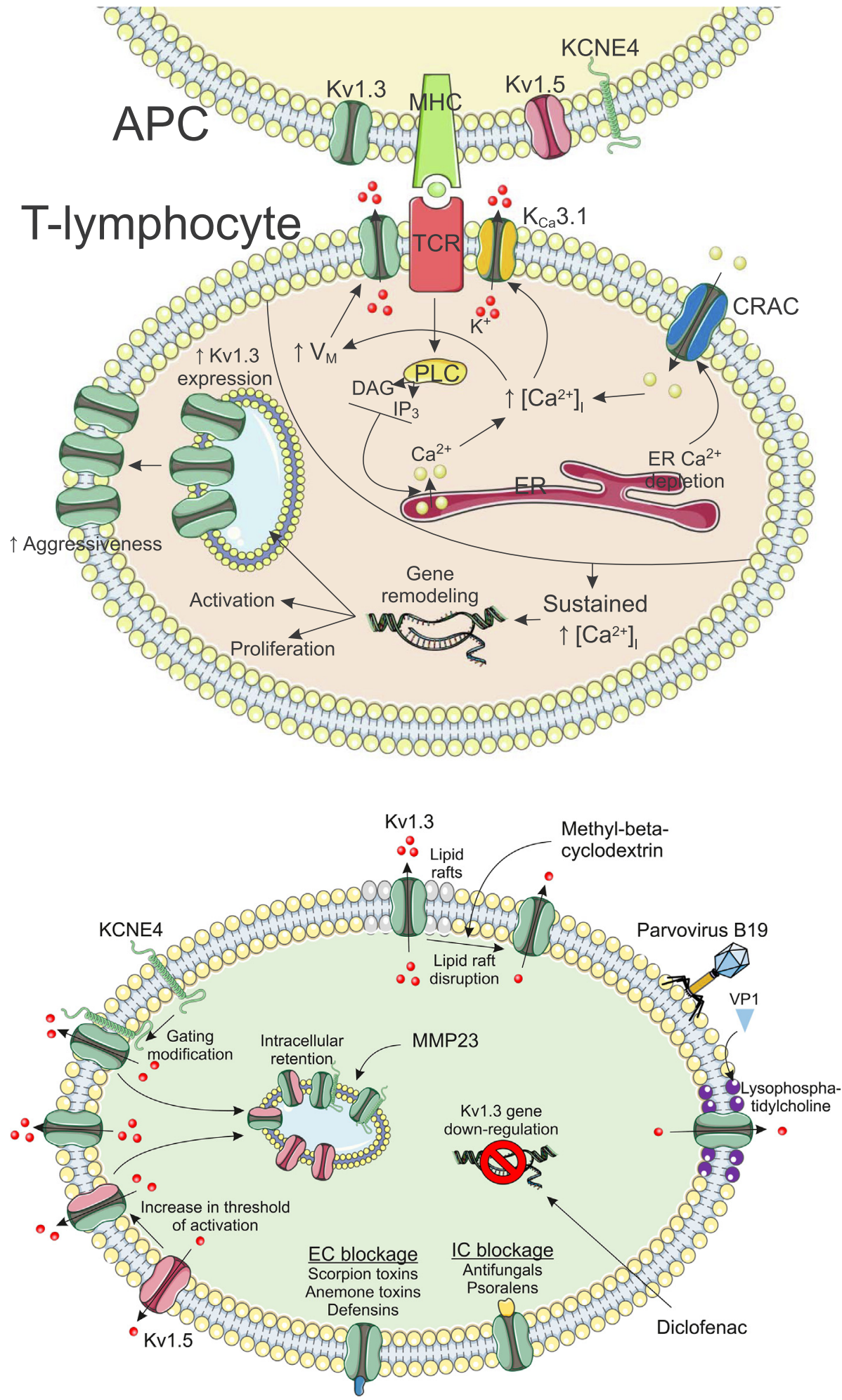

Fig. 1. Summary of $\mathrm{T}$ lymphocyte activation implicating the Kv1.3 channel. T lymphocytes and antigen presenting cells (APC) share Kv1.3 expression. However, APC exhibit a notable amount of Kv1.5 and KCNE4 that further modulate Kv1.3. Antigen presentation by the MHC (major histocompatibility complex)/TCR recognition initiates a signal transduction cascade, which implies the activation of phospholipase C $\gamma$ (PLC). PLC activates diacylglycerol (DAG) and 1,4,5-inositol trisphosphate (IP3) triggering the depletion of internal ER (endoplasmic reticulum) $\mathrm{Ca}^{2+}$ stores. The intracellular $\mathrm{Ca}^{2+}$ augmentation activates calcium release-activated channels (CRAC), which drives more $\mathrm{Ca}^{2+}$ into the cell producing depolarization. Kv1.3 opens by depolarization causing $\mathrm{K}^{+}$efflux that hyperpolarizes the membrane. In addition, the rise in internal $\mathrm{Ca}^{2+}$ concentration $\left[\mathrm{Ca}_{\mathrm{i}}^{2+]}\right.$ activates the intermediateconductance $\mathrm{Ca}^{2+}$-activated $\mathrm{K}^{+}$channel $\left(\mathrm{K}_{\mathrm{Ca}} 3.1\right)$. The synergy of the two $\mathrm{K}^{+}$channels keeps a negative membrane potential adequate for a sustained $\mathrm{Ca}^{2+}$ influx through CRAC channels. Finally, a sustained $\mathrm{Ca}^{2+}$ signal activate nuclear processes, such activation and/or proliferation. An increase of Kv1.3 abundance elevates the response increasing the aggressiveness of the $\mathrm{T}$ cell.
Fig. 2. Mechanisms and consequences of putative Kv1.3-based therapies against autoimmune diseases. Potential Kv1.3 therapies could target directly either the channel or putative associations and spatial locations. The Kv1.3 channelosome is a functional complex that fine-tunes the Kv1.3 activity. Kv1.5, exhibiting differential traffic, targeting and electrophysiological and pharmacological characteristics, heterotetramerizes with Kv1.3. By altering the stoichiometry of the heteromer, the properties of the functional complex differ. KCNE4, which negatively modulates the activity and the surface expression of KCNE4, could be contemplated as a therapeutical target. The Matrix metalloproteinase- 23 also interacts with Kv1.3 retaining the channel at the ER. Animal venoms (toxins) and plant derivatives (psoralens) are quite specific and potent Kv1.3 blockers. Miscellaneous compounds, such as viral molecules (VP1), defensis and antifungals, also show inhibitory activity. Diclofenac, a nonsteroidal anti-inflammatory drug (NSAIDs), impairs the Kv1.3 gene expression. Finally, during the immune system response, Kv1.3 localizes at the immunological synapse between APC and T cell. This synapse concentrates lipid rafts. Controlling this spatial localization, by disrupting rafts with methyl-beta-cyclodextrin, impairs the activity of Kv1.3. IC: Intracellular; EC: Extracellular. See text for further details.
Theoretically, improved hygiene results in lymphocytes being exposed to fewer antigens and are, therefore, overreactive to self-antigens. Neither of these theories has been proven to be the main cause of autoimmune diseases, which suggests that it may be a combination of all of them. Additionally, the existence of familial forms of these diseases and differences between the familial and sporadic forms reveals a genetic implication for pathogenicity [15-17]. Therefore, the pathophysiology of autoimmune diseases shows a complexity comparable to those of cancer processes.

In autoimmune diseases, the voltage-dependent potassium channel Kv1.3 plays a paramount role. T helper lymphocytes activate in contact with an APC (antigen presenting cell), such as macrophages or B lymphocytes (Fig. 1). APC presents the antigen to Thelper cells using MHC II. The detection begins an intracellular signaling cascade. The antigen 
Table 1

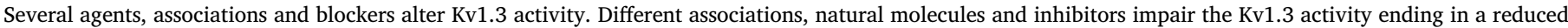

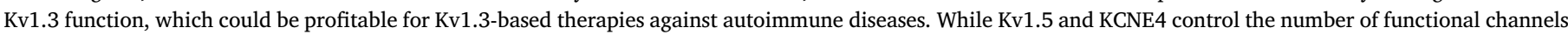

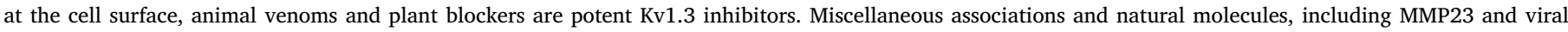

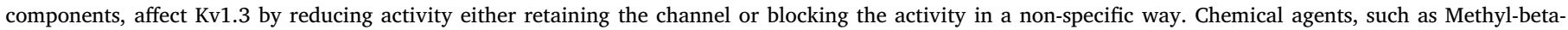
cyclodextrin, disrupt the Kv1.3 signaling platform required for a proper immune response.

\begin{tabular}{|c|c|c|c|}
\hline Targeting mechanism & Modifiers & Mechanism of action & References \\
\hline Heterotetramerization & Kv1.5 & $\begin{array}{l}\text { Intracellular retention, increase in threshold of activation, lipid raft } \\
\text { mistargeting }\end{array}$ & {$[62,108]$} \\
\hline Oligomeric association of the complex & KCNE4 & Intracellular retention, channel gating modification, lipid raft mistargeting & {$[64,65]$} \\
\hline Miscellaneous associations & MMP23 & Intracellular retention & [73] \\
\hline \multirow[t]{2}{*}{ Animal venoms } & $\begin{array}{l}\text { Scorpion toxins (e.g., kaliotoxin, } \\
\text { margatoxin) }\end{array}$ & Extracellular blockage & {$[86,94]$} \\
\hline & Anemone toxins (e.g., ShK) & Extracellular blockage & [95] \\
\hline Plant blockers & Psoralens (e.g., Psora-4, PAP-1) & Intracellular blockage & {$[96,97]$} \\
\hline \multirow[t]{2}{*}{ Miscellaneous Natural } & Antifungals (e.g., nystatin) & Intracellular blockage & [69] \\
\hline & Defensins (e.g., plectasin) & Extracellular blockage & [71] \\
\hline Viral molecules & Virus components (e.g., VP1) & Biochemical downregulation & [75] \\
\hline Chemical agents & Methyl-beta-cyclodextrin & Lipid raft disruption & {$[52-54]$} \\
\hline
\end{tabular}

signal results in the production of $\mathrm{IP}_{3}$ (inositol triphosphate) and DAG (diacylglycerol). At the same time, these molecules spark signaling pathways which elevate the intracellular $\mathrm{Ca}^{2+}$ concentration activating $\mathrm{Ca}^{2+}$-dependent $\mathrm{K}_{\mathrm{Ca}} 3.1$ channels and $\mathrm{Kv} 1.3[18,19]$. Both channels contribute to maintain the $\mathrm{Ca}^{2+}$ influx, which triggers gene remodeling, ending in activation and clonal expansion of lymphocytes (Fig. 1). These channels are essential for the immune activation. While $\mathrm{K}_{\mathrm{Ca}} 3.1$ dominates the immature preactivated $\mathrm{T}$ cells, Kv1.3 is the most important actor in the mature $\mathrm{T}_{\mathrm{EM}}$ cells $[18,20,21]$. $\mathrm{T}_{\mathrm{EM}}$ cells are mature $\mathrm{T}$ lymphocytes which have been exposed repetitively to their antigen. These cells are usually present in peripheric tissues and in circulating blood and lymph [22]. Because $\mathrm{T}_{\mathrm{EM}}$ cells usually reside in inflamed tissues, the effect is very fast facilitating their action in autoimmune diseases once they start to proliferate. Indeed, $\mathrm{T}_{\mathrm{EM}}$ cells with high Kv1.3 expression are found in areas of inflammation related to autoimmune diseases [23]. Therefore, $\mathrm{T}_{\mathrm{EM}}$ and $\mathrm{Kv} 1.3$ could be responsible for the chronicity of autoimmune diseases [19,24]. Evidence demonstrates that Kv1.3-inhibiting drugs also significantly improve the symptoms of autoimmune diseases [25-27].

RA is an autoimmune disorder that mainly targets the synovial joints [28]. Unlike other autoimmune diseases, the trigger for RA is unknown, which hinders its diagnosis and prognosis. Furthermore, several different phenotypes exist, which confirms the heterogeneity of the disease [29]. Even though the pathogenesis is not well understood, RA follows the same pattern as other autoimmune diseases: inflammation, hyperplasia (by swelling) and destruction of the affected tissue, self-antibody production and some systemic effects [28]. The disease focuses on the synovial joints and typical symptoms are synovial inflammation, which progresses to the cartilage, and bone destruction. Because the joint is a structural part of the body, this also implies a certain grade of progressive deformity [28]. In this context, the rheumatoid factor, tightly linked to the disease, is reactive against the Fc portion of the IgG [30]. This interaction provokes a plethora of systemic disorders, such as cardiovascular or pulmonary symptoms, as well as fever [28]. Therefore, RA gradually impairs the motility of patients, which affects them psychologically. This scenario creates a positive feedback loop, which further aggravates the other symptoms [31].

Despite the diversity of autoimmune diseases as well as their chronic and incapacitating nature, the present treatments are limited and suboptimal. The existing first-line medication to treat RA and other autoimmune diseases is methotrexate [32,33], as well as other DMARD (Disease-modifying antirheumatic drugs) such as hydroxychloroquine. Methotrexate is an antimetabolite for the folate molecule which binds to DHFR (dihydrofolate reductase), an enzyme which catalyzes the reaction of dihydrofolate to tetrahydrofolate $[34,35]$. Because this step is necessary for nucleic bases biosynthesis and amino acid processing, methotrexate inhibits the synthesis of DNA, RNA and proteins. Therefore, the antirheumatic function is not selectively targeted but similar to chemotherapy, a collateral side effect of toxicity. Indeed, methotrexate was previously used for the treatment of cancer [36,37]. Although the dose of methotrexate used in RA is lower than the dose used for oncology, long treatment durations often result in adverse effects, such as hair loss, nausea or headaches [38]. Furthermore, methotrexate is a long-known teratogen and abortifacient, which complicates the combination of pregnancy and the disease [39]. Despite these side effects, methotrexate has continued to serve as an essential medicine [40].

Other medications used for the treatment of RA depend on the severity of symptoms and the course of the disease [41]. NSAIDs (Nonsteroidal anti-inflammatory drugs), such as ibuprofen, do help in relieving pain and reducing inflammation. However, a sustained intake can result in gastric damage and compromise cardiovascular safety. In addition, steroids like prednisone have a great anti-inflammatory effect but high doses provoke side effects such as high glycemia or fluid retention [42]. Finally, biologic agents, also known as biologic response modifiers, have been developed. However, biologic agents, targeting the immune system, compromise the immune response elevating the risk of cancer or infections, such as tuberculosis $[43,44]$. The actual choice is DMARD, combined with NSAIDs and steroids, to control pain and inflammation. Biologic agents are only considered once DMARDs fail [45].

Above mentioned therapies are effective to some degree, slowing the course of the disease, but triggering side effects. Therefore, development of new antirheumatic compounds with better therapeutic window and target effectivity deserves more attention.

\section{Kv1.3 as a therapeutic target for autoimmune diseases}

Kv1.3 is a voltage-gated potassium channel which opens at depolarizing membrane potentials [46]. The channel is present in different regions of the CNS (central nervous system), such as the olfactory bulb, and in some nonexcitable tissues, such as adipose tissue or kidneys [47-49]. Regardless, the most essential role of Kv1.3 resides in the immune system. Kv1.3 is expressed in lymphocytes. Kv1.3 is present in the plasma membrane, and targets to the immune synapse during lymphocyte activation [50], but is also detectable in different sub-cellular localizations, such as cis-Golgi, nuclear membrane and mitochondria. However, this review will deal mainly with Kv1.3 located at the cell surface. $\mathrm{T}$ lymphocytes have their TCRs (T-cell receptors) clustered in specific membrane microdomains, called lipid rafts, enriched in cholesterol [51]. The concentration of Kv1.3 in these regions facilitates lymphocyte activation upon an antigen presentation by APCs [52]. In fact, Kv1.3 function is highly dependent on lipid raft location 
$[52,53]$. In fact, the manipulation of membrane lipid composition alters the voltage- and time-dependent gating of the channel [54].

Kv1.3 function is predominant in lymphocyte physiology. Therefore, a connection between Kv1.3 and immune physiology is not surprising. NSAIDs, such as diclofenac, as well as some glucocorticoids, like dexamethasone, reduce Kv1.3 activity in immune cells [55-57]. Moreover, inhibition of Kv1.3 activity impairs the immune response [58]. Thus, pharmacological blockade of Kv1.3 inhibits the proliferation and cytokine production of the pathogenic $\mathrm{T}_{\mathrm{EM}}$ cells while sparing other lymphocyte subpopulations [59]. During the last decade, Kv1.3 blockers have been developed and show a promising effect on autoimmune diseases [60].

In addition to lymphocytes, Kv1.3 is also a predominant channel in the APC of the macrophage lineage [61], also governing cell activation. Furthermore, Kv1.3 abundance correlates with the activation of macrophages. Thus, LPS (lipopolysaccharide)-dependent activation of macrophages increases Kv1.3 expression, while glucocorticoids, such as dexamethasone, reduces the amount of Kv1.3 [55]. In this scenario, macrophages undergo a molecular remodeling of $\mathrm{Kv}$ isoforms, such as Kv1.5. which heterotetramerizing with Kv1.3 modulates the channel [62]. When Kv1.3 increases, the stoichiometry of functional tetramers favors Kv1.3 and results in channels with molecular properties similar to Kv1.3. In contrast, a decrease in Kv1.3 levels results in heteromers with more Kv1.5 units, recapitulating properties of the Kv1.5 homotetramer [61,62]. In this context, Kv1.5 exhibits less membrane expression and activity, and cells are also less sensitive to physiological membrane potentials, which results in a higher threshold of APC activation. In addition, Kv1.3/Kv1.5 heterotetramers are reluctant to Kv1.3 blockers, such as margatoxin which hinders pharmacological treatments [63]. In this context, only psoralens, such as Psora-4, display similar potency for heteromers Kv1.3/Kv1.5 [48].

Kv1.3 also interacts with the regulatory subunit KCNE4, impairing both membrane localization and activity $[64,65]$. Kv1.3 has notorious membrane expression partly due to an anterograde signature (YMVIEE) located in the C-terminus [66]. This signature interacts with Sec24 and facilitates the forward traffic of Kv1.3 via the COPII machinery. KCNE4 association, masking this signal, impairs the COPII-mediated anterograde transport and the expression of the channel at the plasma membrane [67]. Similar to Kv1.3, KCNE4 is tightly regulated in leukocytes under several insults, which could fine-tune the channel function $[64,65]$. Therefore, KCNE4 should be considered as a potential target, but further research must be conducted.

Kv1.3 functional activity is modulated by a wide repertoire of ancillary peptides and signaling pathways. For example, $\mathrm{Na}_{\mathrm{V}} \mathrm{\beta} 1$, a regulatory subunit for $\mathrm{Na}_{\mathrm{V}}$ channels, also modifies Kv1.3 gating by presumably strengthening the coupling between activation of the voltagesensing domain of the channel and the opening of the pore [68]. In addition, antifungal compounds, such as nystatin and amphotericin B, exert a potent inhibition of Kv1.3 from the cytoplasmic side [69]. However, defensin peptides, such as plectasin, block the channel from the extracellular side $[70,71]$. Sphingomyelin also favors a more electropositive threshold of activation in T-lymphocytes [72]. Therefore, these molecules reduce the Kv1.3 aperture within the same voltages. Furthermore, the pro-domain of MMP23 (matrix metalloprotease 23) forces the intracellular retention of Kv1.3, thereby depleting the membrane of channels [73]. Interestingly, MMP23 is highly expressed in some subtypes of lymphocytes, such as the NK (natural killer) cells [74]. Finally, components of some viruses, such as the capsid protein VP1 of Parvovirus B19, downregulate Kv1.3, as well as other ion channels [75].

As mentioned above, Kv1.3 can be modulated by several agents which use completely different mechanisms. Kv1.3-based therapies are advantageous because unlike other Kv channels, Kv1.3 has minor implications in heart physiology. Thus, one of the most limiting factors in ion channel drug development, which is the modification of heart physiology, should not hinder Kv1.3 blockers [76]. Furthermore, the
Kv1.3 knockout (KO) animal model is viable [77]. Kv1.3 gene deletion surprisingly does not trigger a massive effect on the immune system likely because of a compensatory increase in $\mathrm{Cl}^{-}$currents in lymphocytes. However, Kv1.3 KO mice show a decrease in the severity of autoimmune diseases, as well as a minor prevalence of tissue-specific $T_{\mathrm{EM}}$ cells [78]. Furthermore, the deficiency of Kv1.3 favors the proliferation of $\mathrm{T}$ lymphocytes with suppressive properties instead of $\mathrm{T}_{\mathrm{EM}}$ [79]. These results correlate with several in vivo studies that have demonstrated that genetic ablation or pharmacological inhibition of Kv1.3 greatly diminishes the production of proinflammatory cytokines from microglia in an LPS-directed model [80]. This evidence justifies targeting macrophage, in addition to lymphocytes for the treatment of autoimmune diseases.

Recent work demonstrated that patients with RA also tend to present comorbidities such as diabetes $(\sim 15 \%)$, hypertension $(\sim 40 \%)$, depression $(\sim 15 \%)$ and gastroduodenal ulcer $(\sim 11 \%)$ [81]. Whether these maladies are related to RA is an open debate not covered in this review. RA patients exhibit an increased risk of suffering from certain illnesses; therefore, these maladies should be screened, diagnosed and treated when positive.

Surprisingly, the scope of Kv1.3 function is wider than initially expected. Thus, blockade of Kv1.3 not only ameliorates the prognosis in autoimmune disease models $[27,82]$ but also shows promising results for other physiological processes. The Kv1.3 KO mouse exhibited enhanced odorant ability (thus, the nickname super-smeller mouse) and resistance to diet-induced obesity $[83,84]$. These effects could be a consequence of increases in physical activity and basal metabolic rate, which depend on the olfactory bulb. In wild type animals, a similar effect is triggered by the activation of the insulin receptor in the olfactory bulb, which inhibits Kv1.3 by phosphorylation. Therefore, insulin resistance hinders this process, which explains the positive feedback of symptoms in type 2 diabetes mellitus. Furthermore, insulin resistance normally includes high adiposity, anxiety, and reduction in cognitive abilities [85]. Interestingly, insulin resistance (diabetes) and anxiety (depression) are tightly related with some comorbidities of RA. In this context, some works corroborate that the genetic ablation or inhibition of Kv1.3 improves mood and associative learning in rodents, aside from triggering resistance to diet-induced obesity $[83,86]$. Moreover, proof-of-concept studies have demonstrated the potential use of Kv1.3 blockers to treat other neurological syndromes such as Alzheimer [87]. Therefore, pharmacological blockade of Kv1.3 activity could be beneficial for RA as well as some related disorders.

\section{Kv1.3 in rheumatoid arthritis: the other side of the coin}

Kv1.3 stands out as a potential target for the treatment of RA among the autoimmune disorders. However, controversial results arguing against Kv1.3 have opened the debate.

First, the super-smeller mouse Kv1.3 KO model exhibits minor immune defects [77]. As mentioned above, the reason could be remodeling of the $\mathrm{Cl}^{-}$currents, which could compensate for the deficiency in $\mathrm{K}^{+}$currents in $\mathrm{T}$ lymphocytes. Additionally, murine lymphocytes possess additional $\mathrm{K}^{+}$channels and transporters that could drive $\mathrm{K}^{+}$in the absence of Kv1.3 [88-90]. Such a repertoire of channels generates a great variety of ion channel phenotypes in these cells that could mask the importance of Kv1.3 in rodents. In contrast, human T lymphocytes mainly express Kv1.3, with relatively minor influence from other subunits. Another source of variability is that the human Kv1.3 has an additional $5^{\prime}$ upstream starting codon, which produces a 52-residue longer $\mathrm{N}$-terminal domain. This sequence seems to impair the surface membrane expression of the channel in Xenopus oocytes [68]. This longer domain could trigger differential phenotypes in human $\mathrm{T}$ lymphocytes. No pharmacological studies have been conducted on the longer human Kv1.3 form and Kv1.3 blockers, validated in rodents, could trigger unexpected behaviors in humans. Therefore, additional experiments should be conducted on the longer Kv1.3 human form as 
well as with the primate, pig or fish orthologues, which also possess the additional N-terminal sequence [68]. Finally, additional features for the therapeutic use of Kv1.3 in RA are the non-conducting properties of the channels [91]. In this context, Kv1.3 regulates cell proliferation in the vascular tissue by an ion-flux independent mechanism [92].

\section{Pharmacological development}

In this context, new research aims to develop Kv1.3 blockers, which could be used in the treatment of autoimmune diseases. Kv1.3 is affected by a great collection of natural toxins. Therefore, research has focused on modification, thereby improving the specificity, pharmacokinetics and pharmacodynamics [82,93]. The most well-known natural toxins come from animal venoms, such as scorpions (margatoxin and kaliotoxin) and anemones (ShK) $[86,94,95]$. Other blockers are mainly small molecules (PAP-1, Psora-4, and clofazimine), mostly from plant origin [96,97].

One promising molecule is dalazatide, a modified form of ShK (Stichodactila toxin) [98]. ShK is a 35-residue peptide produced by the sea anemone Stichodactila helianthus, which blocks neuronal and lymphocyte voltage-dependent potassium channels [95]. Because ShK exhibits affinity towards Kv1.3 but also Kv1.1 and Kv1.6 [99], several ShK analogs were developed. For instance, ShK-186 increased the selectivity for Kv1.3 by modifying some amino acids of the structure [100]. Thus, ShK-186, renamed dalazatide, became the first Kv1.3 blocker to be tested on humans [98]. The primary scope for this medication is psoriasis, an autoimmune disease mainly targeting the skin, currently undergoing phase II clinical trials. However, recent results also support the use of dalazatide in other autoimmune diseases such as sporadic inclusion body myositis and lupus nephritis [101,102]. The similar etiology and molecular background of these autoimmune diseases may also broaden the scope of the medication, as changes in the formulation can facilitate use in many different diseases. The pharmacokinetics of dalazatide support lasting treatments, as the medication (ClinicalTrials. gov: NCT02435342) shows little to no accumulation after 29 days [98]. Moreover, dalazatide administration is subcutaneous [98]. In this context, alternative administration routes are being considered by several groups. For instance, chitosan-based gels have been proven to improve the buccal mucosal absorption of ShK [103].

Even though dalazatide is a first-in-class medication using Kv1.3 as target against autoimmune diseases, many advances are continuously advancing this field. Scorpion AnTx (anurotoxin) has been modified to reduce its affinity towards Kv1.2 in favor of Kv1.3 [104], and scorpion Vm24, OsK1 and OdK2 have been fused with an antibody Fc (constant fraction) to increase their plasma half-life $[105,106]$. Pulmonary delivery has also been studied as a noninvasive alternative and has already been successfully demonstrated with an HsTx1 (Heterometrus spinifer toxin) analog [107].

In parallel, Kv1.3 modulation should not be limited to a blockade (Fig. 2). As mentioned above, Kv1.3 heteromerizes with other isoforms, such as Kv1.5; and regulatory subunits such as KCNE4, and the Kv $\beta$ family $[64,108,109]$ in native cells. Interaction with such subunits modifies the activity and threshold of activation of the complex, thus altering the leukocyte activation $[62,63,67]$. Efforts to control the expression of such proteins or to emulate their effects would be highly advisable to modulate the channel with a less binary outcome than blockers.

Therefore, the use of Kv1.3 as a therapeutic target for autoimmune diseases in general and RA in particular is a novel method with huge potential. Combinational therapies for RA deserve further attention $[110,111]$. Specific Kv1.3 blockers could substitute actual medications, such as NSAIDs or steroids, which partially targeting Kv1.3, exhibit harmful side effects [55-57].

Finally, because most autoimmune diseases share an elevated $\mathrm{T}_{\mathrm{EM}}$ population, which tightly depends on Kv1.3, Kv1.3-based therapies could be repurposed and extended further than RA [18,20,101,102].

\section{Concluding remarks}

$\mathrm{RA}$ is an autoimmune disease with serious effects on the wellbeing of patients and social environments. The progression of symptoms greatly impairs the normal life of patients both physically and psychologically. Therefore, the socioeconomic cost of this disease is very high for the health system. Considering that RA etiology shares most features of many autoimmune diseases, the need for an effective treatment deserves more effort.

The current first-line treatment for RA, methotrexate, is effective but carries potential diverse side effects similar to chemotherapy drugs. These harmful consequences sometimes lead to a certain level of treatment discontinuation. In persistent and long-term chronic diseases, the adherence to the treatment may have a marked difference on the wellbeing of the patient. Moreover, as methotrexate is a teratogen, a pregnancy-friendly treatment could help female patients improve their quality of life by preventing the disease from affecting their family planning.

In this context, Kv1.3 is a promising therapeutic target for the treatment of RA and for autoimmune diseases in general (Table 1). Kv1.3 plays a relevant role during immune system responses. This role is even more apparent in the $\mathrm{T}_{\mathrm{EM}}$ cells, which are the main driving force of autoimmune progression. Consequently, the inhibition of Kv1.3 ameliorates autoimmune disease symptoms in animals. Moreover, Kv1.3 stands out as a more specific alternative to treatments such as methotrexate because both the pharmacological inhibition and genetic ablation of the channel in animal models result in no pernicious effects on the animals. Nonetheless, differences between rodent and human lymphocytes are not irrelevant. Human lymphocytes express mainly Kv1.3 as a unique voltage-gated potassium channel, while rodents also possess additional Kv1 isoforms. Additionally, human Kv1.3 possesses an additional upstream methionine codon, which adds 52 amino acids to the protein. These differences could add variability to the development of Kv1.3 blockers and should be considered when transitioning newly developed drugs to the clinical stage.

Taking into account all the limitations mentioned throughout this review, a great amount of resources is being funneled into both basic and clinical research for Kv1.3 as a potential therapeutic agent in several autoimmune diseases including RA.

\section{Acknowledgements}

The work carried out by the Molecular Physiology Laboratory was funded by Ministerio de Economia y Competitividad (Spain) grants (BFU2014-54928-R and BFU2017-87104-R) and Fondo Europeo de Desarrollo Regional (FEDER). The Molecular Physiology Laboratory would like to acknowledge all past members who have contributed to this research. The editorial assistance of the American Journal Experts is also acknowledged.

\section{References}

[1] A.D. Davidson, The Autoimmune Diseases, 5th ed., Elsevier Inc., 2014.

[2] E. Goldmuntz, A.S. Penn, Autoimmune diseases. Office on Women's Health: U.S. Department of Health \& Human Services; 2018 [updated May 22, 2018; cited 2018 November 8, 2018]; Available from: https://www.womenshealth.gov/a-z-topics/ autoimmune-diseases.

[3] D. McAlpine, Multiple sclerosis: a plea for a fresh outlook, Br. Med. J. 1 (5017) (1957) 475-480 Epub 1957/03/02.

[4] M. Harboe, Interactions of rheumatoid factor with immune precipitate containing antibody of human origin, Ann. Rheum. Dis. 20 (1961) 363-368 Epub 1961/ $12 / 01$.

[5] T.E. Piemme, Myasthenia gravis and auto-immune disease: review of the literature including a case report of the coexistence of myasthenia and systemic lupus erythematosus, Ann. Intern. Med. 60 (1964) 130-135 Epub 1964/01/01.

[6] W.P. Faulk, J.H. Karam, H.H. Fudenberg, Human anti-insulin antibodies, J. Immunol. 106 (4) (1971) 1112-1116 Epub 1971/04/01.

[7] M. Shiner, D.H. Shmerling, The immunopathology of coeliac disease, Digestion 5 (2) (1972) 69-88 Epub 1972/01/01.

[8] S.M. Hayter, M.C. Cook, Updated assessment of the prevalence, spectrum and case 
definition of autoimmune disease, Autoimmun. Rev. 11 (10) (2012) 754-765 Epub 2012/03/06.

[9] E. Witebsky, N.R. Rose, K. Terplan, J.R. Paine, R.W. Egan, Chronic thyroiditis and autoimmunization, J. Am. Med. Assoc. 164 (13) (1957) 1439-1447 Epub 1957/ $07 / 27$.

[10] K. Murphy, C. Weaver, Janeway's Immunobiology. 8th ed. Science G, editor 2012.

[11] G. Gammon, E. Sercarz, How some T cells escape tolerance induction, Nature 342 (6246) (1989) 183-185 Epub 1989/11/09.

[12] K.W. Wucherpfennig, J.L. Strominger, Molecular mimicry in T cell-mediated autoimmunity: viral peptides activate human $\mathrm{T}$ cell clones specific for myelin basic protein, Cell 80 (5) (1995) 695-705 Epub 1995/03/10.

[13] E. Maverakis, K. Kim, M. Shimoda, M.E. Gershwin, F. Patel, R. Wilken, et al., Glycans in the immune system and The Altered Glycan Theory of Autoimmunity: a critical review, J. Autoimmun. 57 (2015) 1-13 Epub 2015/01/13.

[14] G.A. Rook, Hygiene hypothesis and autoimmune diseases, Clin. Rev. Allergy Immunol. 42 (1) (2012) 5-15 Epub 2011/11/18.

[15] M.J. Fernandez-Perez, O. Barakat, J.M. Garcia-Moreno, M. Lucas, G. Navarro, J.M. Gata, et al., Clinical features of familial multiple sclerosis in Spain, Rev. Neurol. 29 (8) (1999) 693-696 Epub 1999/11/24. Caracteristicas clinicas de la esclerosis multiple familiar en Espana.

[16] T. Nemes, E. Gyodi, F. Szanyo, K. Rajczy, G. Petranyi, Genetic background of rheumatoid arthritis in connection with a family tree, Orvosi. Hetilap. 142 (50) (2001) 2785-2790 Epub 2002/02/01. A rheumatoid arthritis genetikai hattererol egy csaladfa kapcsan.

[17] A.J. Ruiz-Padilla, J.I. Gamez-Nava, A.M. Saldana-Cruz, J.D. Murillo-Vazquez, M.L. Vazquez-Villegas, S.A. Zavaleta-Muniz, et al., The -174G/C interleukin-6 gene promoter polymorphism as a genetic marker of differences in therapeutic response to methotrexate and leflunomide in rheumatoid arthritis, Biomed. Res. Int. 2016 (2016) 4193538 Epub 2016/10/16

[18] G. Panyi, Biophysical and pharmacological aspects of $\mathrm{K}+$ channels in T lymphocytes, Eur. Biophys. J.: EBJ 34 (6) (2005) 515-529 Epub 2005/09/27.

[19] C. Beeton, K.G. Chandy, Potassium channels, memory T cells, and multiple sclerosis, The Neuroscientist 11 (6) (2005) 550-562 Epub 2005/11/12.

[20] H. Rus, C.A. Pardo, L. Hu, E. Darrah, C. Cudrici, T. Niculescu, et al., The voltagegated potassium channel Kv1.3 is highly expressed on inflammatory infiltrates in multiple sclerosis brain, Proc. Natl. Acad. Sci. U.S.A. 102 (31) (2005) 11094-11099 Epub 2005/07/27.

[21] Z. Varga, P. Hajdu, G. Panyi, Ion channels in T lymphocytes: an update on facts, mechanisms and therapeutic targeting in autoimmune diseases, Immunol. Lett. 130 (1-2) (2010) 19-25 Epub 2009/12/23.

[22] F. Sallusto, D. Lenig, R. Forster, M. Lipp, A. Lanzavecchia, Two subsets of memory T lymphocytes with distinct homing potentials and effector functions, Nature 401 (6754) (1999) 708-712 Epub 1999/10/28.

[23] C. Beeton, H. Wulff, N.E. Standifer, P. Azam, K.M. Mullen, M.W. Pennington, et al., Kv1.3 channels are a therapeutic target for T cell-mediated autoimmune diseases, Proc. Natl. Acad. Sci. U.S.A. 103 (2006) 17414-17419 Epub 2006/11/08.

[24] M.D. Cahalan, K.G. Chandy, The functional network of ion channels in T lymphocytes, Immunol. Rev. 231 (1) (2009) 59-87 Epub 2009/09/17.

[25] C. Beeton, H. Wulff, J. Barbaria, O. Clot-Faybesse, M. Pennington, D. Bernard, et al., Selective blockade of T lymphocyte $\mathrm{K}(+)$ channels ameliorates experimental autoimmune encephalomyelitis, a model for multiple sclerosis, PNAS 98 (24) (2001) 13942-13947 Epub 2001/11/22.

[26] E.J. Tarcha, V. Chi, E.J. Munoz-Elias, D. Bailey, L.M. Londono, S.K. Upadhyay, et al., Durable pharmacological responses from the peptide ShK-186, a specific Kv1.3 channel inhibitor that suppresses T cell mediators of autoimmune disease, $J$. Pharmacol. Experimental Therapeutics 342 (3) (2012) 642-653 Epub 2012/ 05/29.

[27] X.L. Yuan, Y.P. Zhao, J. Huang, J.C. Liu, W.Q. Mao, J. Yin, et al., A Kv1.3 channelspecific blocker alleviates neurological impairment through inhibiting T-cell activation in experimental autoimmune encephalomyelitis, CNS Neurosci. Therapeutics 24 (10) (2018) 967-977 Epub 2018/03/27.

[28] I.B. McInnes, G. Schett, The pathogenesis of rheumatoid arthritis, N. Engl. J. Med. 365 (23) (2011) 2205-2219 Epub 2011/12/14

[29] D. Aletaha, T. Neogi, A.J. Silman, J. Funovits, D.T. Felson, C.O. Bingham 3rdet al., 2010 Rheumatoid arthritis classification criteria: an American College of Rheumatology/European League Against Rheumatism collaborative initiative, Arthritis Rheum. 62 (9) (2010) 2569-2581 Epub 2010/09/28.

[30] W.J. Falkenburg, D. van Schaardenburg, P. Ooijevaar-de Heer, G. Wolbink, $\mathrm{T}$. Rispens, IgG subclass specificity discriminates restricted IgM rheumatoid factor responses from more mature anti-citrullinated protein antibody-associated or isotype-switched IgA responses, Arthritis Rheumatol. 67 (12) (2015) 3124-3134 Epub 2015/08/08.

[31] B.W. Smith, A.J. Zautra, The effects of anxiety and depression on weekly pain in women with arthritis, Pain 138 (2) (2008) 354-361 Epub 2008/02/22.

[32] J. Baum, J. Vaughan, Immunosuppressive drugs in rheumatoid arthritis, Ann. Intern. Med. 71 (1) (1969) 202-204 Epub 1969/07/01.

[33] M.W. Brandriss, Methotrexate: suppression of experimental allergic encephalomyelitis, Science 140 (3563) (1963) 186-187 Epub 1963/04/12.

[34] P.T. Rajagopalan, Z. Zhang, L. McCourt, M. Dwyer, S.J. Benkovic, G.G. Hammes, Interaction of dihydrofolate reductase with methotrexate: ensemble and singlemolecule kinetics, PNAS 99 (21) (2002) 13481-13486 Epub 2002/10/03.

[35] D.S. Goodsell, The molecular perspective: methotrexate, Stem Cells 17 (5) (1999) 314-315 Epub 1999/10/20.

[36] J.F. Holland, Methotrexate therapy of metastatic choriocarcinoma, Am. J. Obstet. Gynecol. 75 (1) (1958) 195-199 Epub 1958/01/01.

[37] R.D. Sullivan, Continuous arterial infusion of methotrexate with intermittent, intramuscular citrovorum factor therapy in head and neck cancer, Trans. Pacific Coast Oto-Ophthalmol. Soc. Annu. Meet. 42 (1961) 29-42 Epub 1961/01/01.

[38] K.C. LaMattina, S.M. Escott, J.F. Malalis, D. Mai, Y. Harada, D.A. Goldstein, The influence of race and sex on the side effect profile of methotrexate in the treatment of uveitis, Ocular Immunol. Inflamm. (2018) 1-5 Epub 2018/08/14.

[39] E. Gromnica-Ihle, K. Kruger, Use of methotrexate in young patients with respect to the reproductive system, Clin. Exp. Rheumatol. 28 (5 Suppl. 61) (2010) S80-S84 Epub 2010/11/26.

[40] WHO Model List of Essential Medicines (20th List). World Health Organization, 2017.

[41] Mayo Clinic's web page. 2019 [cited 2019 05-Mar-2019]; Available from: https:// www.mayoclinic.org/.

[42] M. Del Grossi Moura, L. Cruz Lopes, M.T. Silva, S. Barberato-Filho, R.H.L. Motta, C.C. Bergamaschi, Use of steroid and nonsteroidal anti-inflammatories in the treatment of rheumatoid arthritis: systematic review protocol, Medicine 97 (41) (2018) e12658 Epub 2018/10/14.

[43] D. Hochman, B. Wolff, Risk of serious infections and malignancies with anti-TNF antibody therapy in rheumatoid arthritis, Jama 296 (18) (2006) 2203 author reply -4. Epub 2006/11/09.

[44] E.S. Molloy, L.H. Calabrese, Progressive multifocal leukoencephalopathy associated with immunosuppressive therapy in rheumatic diseases: evolving role of biologic therapies, Arthritis Rheum. 64 (9) (2012) 3043-3051 Epub 2012/03/17.

[45] J.S. Smolen, R. Landewe, F.C. Breedveld, M. Buch, G. Burmester, M. Dougados, et al., EULAR recommendations for the management of rheumatoid arthritis with synthetic and biological disease-modifying antirheumatic drugs: 2013 update, Ann. Rheum. Dis. 73 (3) (2014) 492-509 Epub 2013/10/29.

[46] M.D. Cahalan, K.G. Chandy, T.E. DeCoursey, S. Gupta, A voltage-gated potassium channel in human T lymphocytes, J. Physiol. 358 (1985) 197-237 Epub 1985/ $01 / 01$.

[47] V.R. Gazula, J.G. Strumbos, X. Mei, H. Chen, C. Rahner, L.K. Kaczmarek, Localization of Kv1.3 channels in presynaptic terminals of brainstem auditory neurons. J. Comp. Neurol. 518 (16) (2010) 3205-3220 Epub 2010/06/25.

[48] M. Perez-Verdaguer, J. Capera, M. Ortego-Dominguez, J. Bielanska, N. Comes, R.J. Montoro, et al., Caveolar targeting links Kv1.3 with the insulin-dependent adipocyte physiology, Cell. Mol. Life Sci.: CMLS 75 (21) (2018) 4059-4075 Epub 2018/06/28.

[49] L.I. Escobar, J.C. Martinez-Tellez, M. Salas, S.A. Castilla, R. Carrisoza, D. Tapia, et al., A voltage-gated $\mathrm{K}(+)$ current in renal inner medullary collecting duct cells, Am. J. Physiol. Cell Physiol. 286 (4) (2004) C965-C974 Epub 2003/12/20.

[50] G. Panyi, G. Vamosi, Z. Bacso, M. Bagdany, A. Bodnar, Z. Varga, et al., Kv1.3 potassium channels are localized in the immunological synapse formed between cytotoxic and target cells, Proc. Natl. Acad. Sci. U.S.A. 101 (5) (2004) 1285-1290 Epub 2004/01/28.

[51] J. Dinic, A. Riehl, J. Adler, I. Parmryd, The T cell receptor resides in ordered plasma membrane nanodomains that aggregate upon patching of the receptor, Sci. Rep. 5 (2015) 10082 Epub 2015/05/09.

[52] I.I. Pottosin, G. Valencia-Cruz, E. Bonales-Alatorre, S.N. Shabala, O.R. Dobrovinskaya, Methyl-beta-cyclodextrin reversibly alters the gating of lipid rafts-associated Kv1.3 channels in Jurkat T lymphocytes. Pflugers Archiv: Eur. J. Physiol. 454 (2) (2007) 235-244 Epub 2007/01/24.

[53] M. Perez-Verdaguer, J. Capera, R. Martinez-Marmol, M. Camps, N. Comes, M.M. Tamkun, et al., Caveolin interaction governs Kv1.3 lipid raft targeting, Scientific Rep. 6 (2016) 22453 Epub 2016/03/05.

[54] P. Hajdu, Z. Varga, C. Pieri, G. Panyi, R. Gaspar Jr., Cholesterol modifies the gating of Kv1.3 in human T lymphocytes, Pflugers Archiv: Eur. J. Physiol. 445 (6) (2003) 674-682 Epub 2003/03/13.

[55] N. Villalonga, M. David, J. Bielanska, R. Vicente, N. Comes, C. Valenzuela, et al. Immunomodulation of voltage-dependent $\mathrm{K}+$ channels in macrophages: molecular and biophysical consequences, J. General Physiol. 135 (2) (2010) 135-147 Epub 2010/01/27.

[56] A. Lampert, M.M. Muller, S. Berchtold, K.S. Lang, M. Palmada, O. Dobrovinskaya, et al., Effect of dexamethasone on voltage-gated $\mathrm{K}+$ channels in Jurkat T-lymphocytes, Pflugers Archiv: Eur. J. Physiol. 447 (2) (2003) 168-174 Epub 2003/ 08/09.

[57] N. Villalonga, M. David, J. Bielanska, T. Gonzalez, D. Parra, C. Soler, et al., Immunomodulatory effects of diclofenac in leukocytes through the targeting of Kv1.3 voltage-dependent potassium channels, Biochem. Pharmacol. 80 (6) (2010) 858-866 Epub 2010/05/22.

[58] G.C. Koo, J.T. Blake, A. Talento, M. Nouyen, S. Lin, A. Sirotina, et al., Blockade of the voltage-gated potassium channel Kv1.3 inhibits immune responses in vivo, J. Immunol. 158 (11) (1997) 5120-5128 Epub 1997/06/01.

[59] L. Hu, M. Pennington, Q. Jiang, K.A. Whartenby, P.A. Calabresi, Characterization of the functional properties of the voltage-gated potassium channel Kv1.3 in human CD4 + T lymphocytes, J. Immunol. 179 (7) (2007) 4563-4570 Epub 2007/ $09 / 20$.

[60] A. Schmitz, A. Sankaranarayanan, P. Azam, K. Schmidt-Lassen, D. Homerick, W. Hansel, et al., Design of PAP-1, a selective small molecule Kv1.3 blocker, for the suppression of effector memory T cells in autoimmune diseases, Mol Pharmacol. 68 (5) (2005) 1254-1270 Epub 2005/08/16.

[61] R. Vicente, A. Escalada, M. Coma, G. Fuster, E. Sanchez-Tillo, C. Lopez-Iglesias, et al., Differential voltage-dependent $\mathrm{K}+$ channel responses during proliferation and activation in macrophages, J. Biol. Chem. 278 (47) (2003) 46307-46320 Epub 2003/08/19.

[62] R. Vicente, A. Escalada, N. Villalonga, L. Texido, M. Roura-Ferrer, M. Martin Satue, et al., Association of Kv1.5 and Kv1.3 contributes to the major voltagedependent K+ channel in macrophages, J. Biol. Chem. 281 (49) (2006) 
37675-37685 Epub 2006/10/14.

63] N. Villalonga, A. Escalada, R. Vicente, E. Sanchez-Tillo, A. Celada, C. Solsona, et al., Kv1.3/Kv1.5 heteromeric channels compromise pharmacological responses in macrophages, Biochem. Biophys. Res. Commun. 352 (4) (2007) 913-918 Epub 2006/12/13.

[64] L. Sole, M. Roura-Ferrer, M. Perez-Verdaguer, A. Oliveras, M. Calvo, J.M. Fernandez-Fernandez, et al., KCNE4 suppresses Kv1.3 currents by modulating trafficking, surface expression and channel gating, J. Cell Sci. 122 (Pt 20) (2009) 3738-3748 Epub 2009/09/24.

[65] L. Sole, A. Vallejo-Gracia, S.R. Roig, A. Serrano-Albarras, L. Marruecos, J. Manils, et al., KCNE gene expression is dependent on the proliferation and mode of activation of leukocytes, Channels (Austin) 7 (2) (2013) 85-96 Epub 2013/01/19.

[66] R. Martinez-Marmol, M. Perez-Verdaguer, S.R. Roig, A. Vallejo-Gracia, P. Gotsi, A. Serrano-Albarras, et al., A non-canonical di-acidic signal at the C-terminus of Kv1.3 determines anterograde trafficking and surface expression, J. Cell Sci. 126 (Pt 24) (2013) 5681-5691 Epub 2013/10/23.

[67] L. Sole, S.R. Roig, A. Vallejo-Gracia, A. Serrano-Albarras, R. Martinez-Marmol, M.M. Tamkun, et al., The C-terminal domain of Kv1.3 regulates functional interactions with the KCNE4 subunit, J. Cell Sci. 129 (22) (2016) 4265-4277 Epub 2016/11/02.

[68] T. Kubota, A.M. Correa, F. Bezanilla, Mechanism of functional interaction between potassium channel Kv1.3 and sodium channel NavBeta1 subunit, Scientific Rep. 7 (2017) 45310 Epub 2017/03/30.

[69] S.J. Hahn, L.Y. Wang, L.K. Kaczmarek, Inhibition by nystatin of Kv1.3 channels expressed in Chinese hamster ovary cells, Neuropharmacology 35 (7) (1996) 895-901 Epub 1996/01/01.

[70] Z. Xie, J. Feng, W. Yang, F. Xiang, F. Yang, Y. Zhao, et al., Human alpha-defensins are immune-related Kv1.3 channel inhibitors: new support for their roles in adaptive immunity, FASEB J. 29 (10) (2015) 4324-4333 Epub 2015/07/08.

[71] F. Xiang, Z. Xie, J. Feng, W. Yang, Z. Cao, W. Li, et al., Plectasin, first animal toxinlike fungal defensin blocking potassium channels through recognizing channel pore region, Toxins 7 (1) (2015) 34-42 Epub 2015/01/09.

[72] D.J. Combs, H.G. Shin, Y. Xu, Y. Ramu, Z. Lu, Tuning voltage-gated channel activity and cellular excitability with a sphingomyelinase, J. Gen. Physiol. 142 (4) (2013) 367-380 Epub 2013/09/18.

[73] H.M. Nguyen, C.A. Galea, G. Schmunk, B.J. Smith, R.A. Edwards, R.S. Norton, et al., Intracellular trafficking of the KV1.3 potassium channel is regulated by the prodomain of a matrix metalloprotease, J. Biol. Chem. 288 (9) (2013) 6451-6464 Epub 2013/01/10.

[74] A.I. Su, T. Wiltshire, S. Batalov, H. Lapp, K.A. Ching, D. Block, et al., A gene atlas of the mouse and human protein-encoding transcriptomes, PNAS 101 (16) (2004) 6062-6067 Epub 2004/04/13.

[75] M. Ahmed, A. Almilaji, C. Munoz, B. Elvira, E. Shumilina, C.T. Bock, et al., Downregulation of $\mathrm{K}(+)$ channels by human parvovirus B19 capsid protein VP1, Biochem. Biophys. Res. Commun. 450 (4) (2014) 1396-1401 Epub 2014/07/11.

[76] S7B: The Non-Clinical Evaluation of the Potential for Delayed Ventricular Repolarization (QT Interval Prolongation) by Human Pharmaceuticals, (2005).

[77] P.A. Koni, R. Khanna, M.C. Chang, M.D. Tang, L.K. Kaczmarek, L.C. Schlichter, et al., Compensatory anion currents in Kv1.3 channel-deficient thymocytes, J. Biol. Chem. 278 (41) (2003) 39443-39451 Epub 2003/07/25.

[78] A.R. Gocke, L.A. Lebson, I.V. Grishkan, L. Hu, H.M. Nguyen, K.A. Whartenby, et al., Kv1.3 deletion biases T cells toward an immunoregulatory phenotype and renders mice resistant to autoimmune encephalomyelitis, J. Immunol. 188 (12) (2012) 5877-5886 Epub 2012/05/15

[79] I.V. Grishkan, D.M. Tosi, M.D. Bowman, M. Harary, P.A. Calabresi, A.R. Gocke, Antigenic stimulation of Kv1.3-deficient th cells gives rise to a population of Foxp3-independent T cells with suppressive properties, J. Immunol. 195 (4) (2015) 1399-1407 Epub 2015/07/08.

[80] J. Di Lucente, H.M. Nguyen, H. Wulff, Jin LW, Maezawa I. The voltage-gated potassium channel Kv1.3 is required for microglial pro-inflammatory activation in vivo, Glia 66 (9) (2018) 1881-1895 Epub 2018/07/26.

[81] M. Dougados, M. Soubrier, A. Antunez, P. Balint, A. Balsa, M.H. Buch, et al., Prevalence of comorbidities in rheumatoid arthritis and evaluation of their monitoring: results of an international, cross-sectional study (COMORA), Ann. Rheum. Dis. 73 (1) (2014) 62-68 Epub 2013/10/08.

[82] A. Ueyama, K. Imura, E. Kasai-Yamamoto, N. Tai, M. Nagira, M. Shichijo, et al. Kv1.3 blockers ameliorate allergic contact dermatitis by preferentially suppressing effector memory T cells in a rat model, Clin. Exp. Dermatol. 38 (8) (2013) 897-903 Epub 2013/11/21.

[83] D.A. Fadool, K. Tucker, R. Perkins, G. Fasciani, R.N. Thompson, A.D. Parsons, et al., Kv1.3 channel gene-targeted deletion produces "Super-Smeller Mice" with altered glomeruli, interacting scaffolding proteins, and biophysics, Neuron 41 (3) (2004) 389-404 Epub 2004/02/10.

[84] K. Tucker, J.M. Overton, D.A. Fadool, Diet-induced obesity resistance of Kv1.3-/mice is olfactory bulb dependent, J. Neuroendocrinol. 24 (8) (2012) 1087-1095 Epub 2012/03/23.

[85] P. Das, A.D. Parsons, J. Scarborough, J. Hoffman, J. Wilson, R.N. Thompson, et al., Electrophysiological and behavioral phenotype of insulin receptor defective mice, Physiol. Behav. 86 (3) (2005) 287-296 Epub 2005/09/24.

[86] S. Kourrich, C. Mourre, B. Soumireu-Mourat, Kaliotoxin, a Kv1.1 and Kv1.3 channel blocker, improves associative learning in rats, Behav. Brain Res. 120 (1) (2001) 35-46 Epub 2001/02/15.

[87] I. Maezawa, H.M. Nguyen, J. Di Lucente, D.P. Jenkins, V. Singh, S. Hilt, et al., Kv1.3 inhibition as a potential microglia-targeted therapy for Alzheimer's disease: preclinical proof of concept, Brain 141 (2) (2018) 596-612 Epub 2017/12/23.
[88] S. Grissmer, S. Ghanshani, B. Dethlefs, J.D. McPherson, J.J. Wasmuth, G.A. Gutman, et al., The Shaw-related potassium channel gene, Kv3.1, on human chromosome 11, encodes the type $1 \mathrm{~K}+$ channel in T cells, J. Biol. Chem. 267 (29) (1992) 20971-20979 Epub 1992/10/15.

[89] Q.H. Liu, B.K. Fleischmann, B. Hondowicz, C.C. Maier, L.A. Turka, K. Yui, et al., Modulation of Kv channel expression and function by TCR and costimulatory signals during peripheral CD4( + ) lymphocyte differentiation, J. Exp. Med. 196 (7) (2002) 897-909 Epub 2002/10/09.

[90] Y. Ishida, T.M. Chused, Lack of voltage sensitive potassium channels and generation of membrane potential by sodium potassium ATPase in murine T lymphocytes, J. Immunol. 151 (2) (1993) 610-620 Epub 1993/07/15.

[91] L.K. Kaczmarek, Non-conducting functions of voltage-gated ion channels, Nat. Rev. Neurosci. 7 (10) (2006) 761-771 Epub 2006/09/22.

[92] P. Cidad, L. Jimenez-Perez, D. Garcia-Arribas, E. Miguel-Velado, S. Tajada, C. Ruiz-McDavitt, et al., Kv1.3 channels can modulate cell proliferation during phenotypic switch by an ion-flux independent mechanism, Arteriosclerosis Thrombosis Vasc. Biol. 32 (5) (2012) 1299-1307 Epub 2012/03/03.

[93] S. Koshy, R. Huq, M.R. Tanner, M.A. Atik, P.C. Porter, F.S. Khan, et al., Blocking KV1.3 channels inhibits Th2 lymphocyte function and treats a rat model of asthma, J. Biol. Chem. 289 (18) (2014) 12623-12632 Epub 2014/03/20.

[94] M. Garcia-Calvo, R.J. Leonard, J. Novick, S.P. Stevens, W. Schmalhofer, G.J. Kaczorowski, et al., Purification, characterization, and biosynthesis of margatoxin, a component of Centruroides margaritatus venom that selectively inhibits voltage-dependent potassium channels, J. Biol. Chem. 268 (25) (1993) 18866-18874 Epub 1993/09/05.

[95] M.W. Pennington, M.E. Byrnes, I. Zaydenberg, I. Khaytin, J. de Chastonay, D.S. Krafte, et al., Chemical synthesis and characterization of ShK toxin: a potent potassium channel inhibitor from a sea anemone, Int. J. Pept. Protein Res. 46 (5) (1995) 354-358 Epub 1995/11/01.

[96] S. Kundu-Raychaudhuri, Y.J. Chen, H. Wulff, Raychaudhuri SP. Kv1.3 in psoriatic disease: PAP-1, a small molecule inhibitor of Kv1.3 is effective in the SCID mouse psoriasis-xenograft model, J. Autoimmun. 55 (2014) 63-72 Epub 2014/09/02.

[97] J. Vennekamp, H. Wulff, C. Beeton, P.A. Calabresi, S. Grissmer, W. Hansel, et al., Kv1.3-blocking 5-phenylalkoxypsoralens: a new class of immunomodulators, Mol Pharmacol. 65 (6) (2004) 1364-1374 Epub 2004/05/25.

[98] E.J. Tarcha, C.M. Olsen, P. Probst, D. Peckham, E.J. Munoz-Elias, J.G. Kruger, et al., Safety and pharmacodynamics of dalazatide, a Kv1.3 channel inhibitor, in the treatment of plaque psoriasis: a randomized phase $1 \mathrm{~b}$ trial, PloS One 12 (7) (2017) Epub 2017/07/21.

[99] K. Kalman, M.W. Pennington, M.D. Lanigan, A. Nguyen, H. Rauer, V. Mahnir, et al., ShK-Dap22, a potent Kv1.3-specific immunosuppressive polypeptide, J. Biol. Chem. 273 (49) (1998) 32697-32707 Epub 1998/11/26.

[100] V. Chi, M.W. Pennington, R.S. Norton, E.J. Tarcha, L.M. Londono, B. Sims-Fahey, et al., Development of a sea anemone toxin as an immunomodulator for therapy of autoimmune diseases, Toxicon 59 (4) (2012) 529-546 Epub 2011/08/27.

[101] T.W. Mozaffar, N.A. Goyal, C. Philips, C. Olsen, K. Munoz, A. Mannaa, A. Villalta, Poster: Kv1.3 expression on effector memory T-cells in sporadic inclusion body myositis: Potential for targeted immunotherapy with Dalazatide, Neuromuscul. Disord. (2018) 27.

[102] A.H. Stevens, M. Yuasa, D. Peckham, C. Olsen, C. Philips, S. Iadonato, P. Probst, Kv1.3 expression on urinary leukocytes in lupus nephritis: potential for targeted immunotherapy, Pediatric Rheumatol. Symposium Houston (2017).

[103] T. Caon, L. Jin, C.M. Simoes, R.S. Norton, J.A. Nicolazzo, Enhancing the buccal mucosal delivery of peptide and protein therapeutics, Pharm. Res. 32 (1) (2015) 1-21 Epub 2014/08/30.

[104] A. Bartok, K. Feher, A. Bodor, K. Rakosi, G.K. Toth, K.E. Kover, et al., An engineered scorpion toxin analogue with improved Kv1.3 selectivity displays reduced conformational flexibility, Scientific Rep. 5 (2015) 18397 Epub 2015/ $12 / 23$.

[105] W. Edwards, W.P. Fung-Leung, C. Huang, E. Chi, N. Wu, Y. Liu, et al., Targeting the ion channel Kv1.3 with scorpion venom peptides engineered for potency, selectivity, and half-life, J. Biol. Chem. 289 (33) (2014) 22704-22714 Epub 2014/ 06/19.

[106] R.E. Wang, Y. Wang, Y. Zhang, C. Gabrelow, V. Chi, Q. Fu, et al., Rational design of a Kv1.3 channel-blocking antibody as a selective immunosuppressant, Proc. Natl Acad. Sci. U.S.A. 113 (41) (2016) 11501-11506 Epub 2016/09/25.

[107] L. Jin, Q.T. Zhou, H.K. Chan, I.C. Larson, M.W. Pennington, R.A.V. Morales, et al., Pulmonary delivery of the Kv1.3-blocking peptide HsTX1[R14A] for the treatment of autoimmune diseases, J. Pharm. Sci. 105 (2) (2016) 650-656 Epub 2016/ $02 / 13$.

[108] R. Vicente, N. Villalonga, M. Calvo, A. Escalada, C. Solsona, C. Soler, et al., Kv1.5 association modifies Kv1.3 traffic and membrane localization, J. Biol. Chem. 283 (13) (2008) 8756-8764 Epub 2008/01/26.

[109] R. Vicente, A. Escalada, C. Soler, M. Grande, A. Celada, M.M. Tamkun, et al., Pattern of Kv beta subunit expression in macrophages depends upon proliferation and the mode of activation, J. Immunol. 174 (8) (2005) 4736-4744 Epub 2005/ 04/09.

[110] Y. Wang, Z. Liu, T. Li, L. Chen, J. Lyu, C. Li, et al., Enhanced therapeutic effect of RGD-modified polymeric micelles loaded with low-dose methotrexate and nimesulide on rheumatoid arthritis, Theranostics 9 (3) (2019) 708-720 Epub 2019/ $02 / 28$.

[111] A. Katri, A. Dabrowska, H. Lofvall, M. Ding, M.A. Karsdal, K.V. Andreassen, et al., Combining naproxen and a dual amylin and calcitonin receptor agonist improves pain and structural outcomes in the collagen-induced arthritis rat model, Arthritis Res. Therapy 21 (1) (2019) 68 Epub 2019/02/24. 